\title{
Teste de inteligência WISC-III Adaptando para a população brasileira ${ }^{1}$
}

\author{
Vera L. M. Figueiredo ${ }^{2}$ e Sílvia Pinheiro \\ Universidade Católica de Pelotas (UCPe/) \\ Elizabeth do Nascimento \\ Universidade Federal de Minas Gerais (UFMG)
}

\begin{abstract}
Resumo
O presente artigo tem por objetivo mostrar alguns resultados encontrados até o momento, na pesquisa de adaptação do teste de inteligência WISC- III (Wechsler Intelligence Scale for ChildrenThird Edition). Os dados apresentados referem-se à aplicação do conjunto verbal do teste a uma amostra piloto de 116 crianças com idades entre 6 e 16 anos, matriculadas na rede de escolas públicas e privadas da cidade do Rio Grande (RS). Os resultados evidenciaram índices de validade e consistência interna do conjunto verbal do teste. Observou-se a relevância da idade, da repetência e do local da escola devendo estas variáveis serem controladas na pesquisa de adaptação. Há necessidade de alteração de alguns itens, principalmente nos subtestes Informação e Vocabulário.
\end{abstract}

Palavras-chaves: WISC-III; teste de inteligência; adaptação.

\section{Intelligence Test WISC - III adaptation to Brazillan population}

\section{Summary}

This article shows some ofthe data ofthe adaptation forthe Brazilian population ofthe intelligence test WISCIII (Wechsler Intelligence Scale for Children-Third Edition) found until the present moment. The data shown are the results of the verbal subtest ofthe WISC-III put into practice with a initial population constituted by 116 children 6 to 16 year-old. They were attending public and private schools of the Rio Grande tawn in Rio Grande do Sul State, Brazil. The results, showed internal validity and consistency. Considering the population, age, school failure and location of the school were aspects that had influence over the results. These variables should be controlled during the following research. The analisys indicated the need ofaltering some ofthem. specially in the subtests Information and Vocabulary.

Key words: WISC-III; Intelligence test; adaptation.

Wechsler, em 1939 (apud Zachary, 1990 p. 277), definiu a inteligência como "uma agregação da capacidade global do indivíduo para agir intencionalmente, para pensar racionalmente e para inserir-se efetivamente no seu meio social", caracterizando o comportamento individual como um todo e não apenas em habilidades específicas. Essa capacidade seria o produto tanto da constituição genética como do esforço, motivação, tendências da personalidade e experiências socioeducacionais do indivíduo. A inteligência é concebida não como uma habilidade particular nem como a soma de habilidades específicas, mas como uma entidade global que será evidenciada pela maneira como o indivíduo, particularmente, associa as habilidades e as aplica na situação prática (Wechsler, 1991; Zachary, 1990; Safra, 1987; Matarazzo, 1976).

\footnotetext{
${ }^{1}$ Pesquisa financiada pela VCPel e CNPq

${ }^{2}$ Endereço para cOlTespondência: SQN. 412 81. 8 Ap. 205, CEP 70867-020. 8rasília - DF, E-mail: veratig@tba.com.br
} 
Para Wechsler, embora a inteligência não seja uma simples soma de aptidões intelectuais, o único meio de poder avaliá-la quantitativamente seria medir os diferentes aspectos dessa aptidão e, dentro dessa perspectiva, elaborou um instrumento de avaliação organizando as questões por tipo de tarefa e especificando os subtestes por dificuldade, contrapondo-se à tradição de agrupar as questões por níveis de idade como se caracterizavam os testes de Binet. As Escalas de Wechsler constituem, no plano da padronização, um aperfeiçoamento sensível em relação ao seu precursor, o teste de Binet e Simon (1905) e aos que dele se derivam. Nas provas de Binet, os subtestes são agrupados e diferenciados por níveis de idade e o QI medido não permite comparar os resultados de um determinado sujeito em idades diferentes, uma vez que com os resultados da prova calcula-se o nível mental do sujeito que é, então, comparado com a idade cronológica e, assim, calculado o QI (Gibello, 1986).

Segundo Zimmerman (1977), as Escalas de Wechsler figuram entre as técnicas que se propõem avaliar a inteligência como as mais bem elaboradas e tipificadas, sendo as de maior fidedignidade e validade copiosamente demonstrada. Ainda, segundo o autor, elas ocupam um ponto referencial na psicologia contemporânea, demonstrando serem úteis para a medida do nível mental e para a interpretação clínica das possibilidades e limitações intelectuais da pessoa. Sua principal relevância consiste em explorar uma amostra muito ampla de funções cognitivas, cuja relativa covariação e cujo progresso cronológico justificam a obtenção de uma medida geral da inteligência.

As Escalas de Wechsler são reconhecidas mundialmente e utilizadas nas mais diversas áreas da prática psicológica, educacional e médica. Em levantamento realizado por Lubin (apud Hutz e Bandeira, 1993), na década de 80, entre os 10 testes mais utilizados na prática profissional, nos Estados Unidos, encontravam-se o WAIS e o WISC-R colocados, respectivamente, em $2^{\circ}$ e $6^{\circ}$ lugar. Almeida (1996) cita a pesquisa de Oakland e Hu, de 1992, que tomou as respostas de psicólogos de 44 países e verificou que entre os 10 testes mais usados internacionalmente, junto a crianças e jovens, 4 deles dizem respeito a testes de inteligência geral encontrando-se, entre eles, o WISC e o WISC-R em I a lugar e o WAIS e o WAIS-R em $6^{\circ}$ lugar.

No Brasil, as Escalas de Wechsler são amplamente utilizadas, porém nenhum trabalho completo de adaptação foi realizado. Em relação à Escala para avaliação da inteligência para adultos (WAIS - Wechsler Adult Intelligence Scale), encontra-se material relacionado à primeira edição do teste (1955), consistindo na tradução de alguns subtestes verbais e na folha para registro de respostas que é distribuída pela editora CEPA (Centro de Editores de Psicologia Aplicada). Em 1997, nos Estados Unidos, foi editada sua terceira edição (WAIS-IIl), que está sendo objeto de pesquisa de adaptação e validação para o contexto brasileiro, por Nascimento (no prelo). Até o momento, não existe nenhuma edição brasileira do referido teste, podendo ser obtido apenas mediante importação. A escala para crianças (WISC - Wechsler Intelligence Scale for Children), teve sua primeira edição (1949) traduzida para a língua nacional por Ana Maria Poppovic, em 1964. $\mathrm{Na}$ tradução foram introduzidas pequenas modificações e adaptações, não sendo, entretanto, estabelecidas normas brasileiras. Este material é editado, também, pelo CEPA.

A única iniciativa na tentativa de minimizar a limitação na utilização do WISC, instrumento simplesmente traduzido para nossa língua, foi o trabalho de adaptação da escala verbal do referido teste realizado por Paine e Lemgruber $(1978,1981)$. A pesquisa foi realizada em 1974, na UFRJ e intitulou-se "Adaptação brasileira da escala verbal do WISC'. O trabalho teve por objetivo revisar, 
adaptar e normatizar os subtestes da escala verbal do referido teste e os resultados foram divulgados através das revistas American Journal of Psychology (1978) e Arquivos Brasileiros de Psicologia (1981). Segundo as próprias autoras, a amostra utilizada para a padronização do teste não pode ser considerada representativa da população brasileira, uma vez que ela incluiu somente crianças que moravam no antigo Estado da Guanabara, devendo ser utilizada com precaução para a criança brasileira.

O WISC-III, editado em 1991, pela Psychological Corporation, é obtido por meio da importação do material original ou da versão argentina, publicada pela Paidós, consistindo esta numa tradução do original americano. A editora Casa do Psicólogo tentou adquirir os direitos autorais do WISC-III, que está sendo reivindicado, entretanto, pelo CEP A que tem os direitos nacionais sobre o WISC - primeira edição do teste. Atualmente, essa questão está sendo resolvida em termos jurídicos e, até sua definição, a comunidade científica fica privada de ter à sua disposição o instrumento na versão nacional.

O referido teste, embora apresente numerosos melhoramentos e substancial número de itens adicionados, mantém as características básicas do WISC e do WISC-R. Mais de $73 \%$ dos itens do WISC-R (não incluindo o subteste Códigos) foram mantidos na forma original ou tiveram mudanças insignificantes. $\mathrm{O}$ teste apresenta 13 subtestes que medem diferentes habilidades da inteligência e são agrupados, como nas demais Escalas de Inteligência de Wechsler, num Conjunto Verbal (Informação; Semelhanças; Vocabulário; Compreensão; Aritmética; Dígitos) e num Conjunto de Execução (Completar Figuras; Arranjo de Figuras; Armar Objetos; Códigos; Cubos; Procurar Símbolos; Labirinto), definindo os QI Verbal, QI de Execução e QI Total.

Adicionalmente, o teste proporciona, ao examinador, quatro índices opcionais denominados índices fatoriais que avaliam a compreensão verbal, a organização perceptual, a resistência à distração e a velocidade de processamento. Tais índices são obtidos por intermédio dos escores nos respectivos subtestes que formam cada fator, conforme ilustrado na tabela 1.

Tabela 1. Índices fatoriais presentes no teste WJSC-III

\begin{tabular}{cccc}
\hline $\begin{array}{c}\text { Fator I } \\
\text { Compreensão } \\
\text { Verbal }\end{array}$ & $\begin{array}{c}\text { Fator II } \\
\text { Organização } \\
\text { perceptual }\end{array}$ & $\begin{array}{c}\text { Fator III } \\
\text { Resistência } \\
\text { à distração }\end{array}$ & $\begin{array}{c}\text { Fator IV } \\
\text { Velocidade de } \\
\text { processamento }\end{array}$ \\
\hline Informação & Completar Figuras & Aritmética & Códigos \\
Semelhanças & Arranjo de Figuras & Dígitos & Procurar símbolos \\
Vocabulário & Cubos & \\
Compreensão & Armar Objetos & \\
\hline \multicolumn{3}{c}{ Fonte: Psychological Corporation } \\
\end{tabular}

Desde a publicação do WISC-III, em 1991, inúmeras pesquisas têm sido feitas utilizando o teste. Os trabalhos referem-se a análises relativas às suas qualidades psicométricas, aos diferentes usos nas áreas da psicologia, da educação e da saúde e à investigação do poder diagnóstico de diferentes quadros patológicos. Em levantamento realizado sobre a produção científica nacional e estrangeira registrada nos bancos de dados informatizados (Psyclit, Eric, Rede Antares e Proquest), encontrou-se, até 1997, um total de 182 referências de trabalhos que utilizaram o referido 
instrumento, sendo 113 em artigos de periódicos e 60 em dissertações e testes de pós-graduação.

Tendo em vista a relevância do WISC-III e na expectativa de adequar uma escala de avaliação intelectual para o país, a pesquisadora iniciou a adaptação desse instrumento, por ocasião de sua dissertação de mestrado (Figueiredo, 1994). O trabalho consistiu na tradução do material original e na aplicação do conjunto verbal do teste a uma amostra de crianças da rede de ensino da cidade de Rio Grande (RS). Considerando que a pesquisa baseou-se num estudo preliminar e parcial das características e da adequação do teste a uma pequena amostra da população brasileira, a proposta da pesquisa está sendo continuada como tese de seu doutoramento e, dessa forma, será possível oferecer aos profissionais da área psi uma técnica de avaliação intelectual atualizada e adequada às características socioeconômicas e culturais de nossa população e com os requisitos psicométricos necessários aos instrumentos de avaliação. A proposta pretende preencher a lacuna referente à falta de instrumentos de avaliação psicológica adaptados e atualizados para nossa realidade que, sem dúvidas, apresenta características próprias e diferenciadas do grupo original de padronização dos instrumentos estrangeiros.

O presente artigo tem por objetivo mostrar os resultados encontrados até o momento, nas etapas já desenvolvidas do processo de adaptação do teste.

\section{Método}

\section{Aplicação do conjunto verbal do teste a uma amostra piloto}

Por ocasião da pesquisa do mestrado, o conjunto de subtestes verbais do WISC-IlI foi aplicado a uma amostra de 116 crianças com idades entre 6 a 16 anos, matriculadas nas escolas da rede pública e privada da cidade de Rio Grande (RS). A aplicação do instrumento foi feita na própria instituição de ensino de cada sujeito, em sessões individuais que duraram em torno de 30 minutos. A forma do teste utilizada na pesquisa constou na tradução do teste original americano WISC-III para a língua portuguesa, incluindo algumas substituições de termos.

\section{Análise teórica dos itens}

Como parte inicial da pesquisa do doutorado procedeu-se a análise teórica dos itens que consiste na análise de juízes e na análise semântica.

A análise de juízes teve como objetivo verificar a pertinência dos itens dos subtestes verbais do WISC-IIl ao constructo que representam, assim como analisar a adequação em relação à formulação das questões e ao nível de conhecimento acadêmico e cultural da população a que o teste se propõe. Participaram desta etapa 08 psicólogos e 16 professores de 10 e 20 graus da rede oficial de ensino da cidade de Pelotas (RS). Foi entregue a eles um questionário acompanhado das definições constitutivas e operacionais de cada subteste para orientar a identificação da pertinência de cada item ao subteste. Após cada questão, apareciam alternativas para serem assinaladas quanto à adequação/inadequação no que se referia à maneira de sua formulação e ao nível de conhecimento da população. No caso de considerar o item inadequado, o juiz deveria sugerir outro para substituílo, respeitando o nível de dificuldade e o objetivo do mesmo.

A análise semântica foi realizada com os propósitos de verificar tanto se os itens do teste estavam compreensíveis para o estrato mais baixo (6-7 anos) da população-meta como para evitar a deselegância na formulação dos itens e garantir a validade aparente do instrumento junto à amostra mais sofisticada (15-16 anos). Para tal, foram entrevistados dois grupos de 12 alunos de escola 
particular e pública da cidade de Pelotas (RS), sendo dois de cada extremo da faixa etária. Os sujeitos foram questionados sobre a compreensão dos itens dos subtestes do conjunto verbal e sobre as instruções de todos os subtestes. As respostas foram gravadas em fita $\mathrm{K} 7 \mathrm{e}$, posteriormente, transcritas para a interpretação dos dados.

\section{Resultados}

\section{Aplicação do conjunto verbal a uma amostra piloto}

a. Dados referentes a parâmetros psicométricos, concluídos a partir de análises sobre a validade e fidedignidade do conjunto verbal do teste:

Há validade interna no conjunto de itens verbais do WISC-II1, considerando os coeficientes significativos obtidos na análise de Correlação de Pearson entre os subtestes (tab. 2) e entre cada subteste e o número total de acertos no conjunto verbal do teste (tab. 3).

Tabela 2. Matriz de correlação entre os subtestes verbais do WISC-l11

\begin{tabular}{lcccccc}
\hline Subteste & Compreensão & Informação & dígitos & Semelhanças & Vocabulário & Aritmética \\
\hline Compreensão & & & & & & \\
Informação & 0,81 & & & & & \\
Dígitos & 0,54 & 0,68 & & & & \\
Semelhenças & 0,77 & 0,88 & 0,69 & & & \\
Vocabulário & 0,83 & 0,92 & 0,70 & 0,87 & 0,81 & \\
Aritmética & 0,75 & 0,84 & 0,72 & 0,82 & \\
\hline
\end{tabular}

$\mathrm{P}<0.0001$ para todas as correlações.

Fonte: Dados da pesquisa Influências socioculturais na inteligência verbal: uma análise fundamentada no teste WISC-1Il, 1994.

Tabela 3. Correlações entre os escores no subtestes e o escore no conjunto verbal total.

\begin{tabular}{c|c}
\hline Subteste & correlação \\
\cline { 2 - 3 } Vocabulário & 0,96 \\
Informação & 0,95 \\
Semelhanças & 0,94 \\
Aritmética & 0,90 \\
Compreensão & 0,88 \\
Dígitos & 0,77 \\
\hline
\end{tabular}

$\mathrm{P}<0.0001$ para todas as correlações.

Fonte: Dados da pesquisa Influências socioculturais na inteligência verbal: uma análise fundamentada no teste WISC-1Il, 1994.

Os dados evidenciam validade interna e presença de um fator comum entre os subtestes 
verbais, tendo em vista o inter-relacionamento entre os subtestes. Observa-se que os maiores índices de correlação encontram-se nos subtestes Vocabulário, Informação e Semelhanças, dados similares aos encontrados na pesquisa de padronização americana do WISC-II1, onde os mesmos subtestes apresentaram, respectivamente, os maiores índices de correlação com o QI Verbal.

Há consistência interna entre os itens verbais do teste WISC-II1, tendo em vista os índices de fidedignidade obtidos através do alfa de Cronbach, conforme tab. 4.

Tabela 4. Coeficientes de consistência interna dos subtestes (alfa)

\begin{tabular}{ll}
\hline Subteste & Alfa \\
\cline { 2 - 2 } Informação & 0,93 \\
Vocabulário & 0,91 \\
Semelhanças & 0,89 \\
Aritmética & 0,97 \\
Compreensão & 0,83 \\
Dígitos & 0,79 \\
\hline
\end{tabular}

$\alpha$ Total $=0,94$

Fonte: Dados da pesquisa Influências socioculturais na inteligência verbal: uma análise fundamentada no teste W1SC-llJ. 1994.

Os valores de alfa evidenciam precisão do conjunto verbal do teste WISC-l1l. O subteste Dígitos parece ser o menos fidedigno para avaliar a habilidade intelectual da amostra, dado semelhante ao obtido no processo de padronização americana que, no cálculo da fidedignidade, determinado pelo Método do Teste / Reteste, evidenciou o menor coeficiente de precisão no mesmo subteste.

b. Dados referentes à variáveis que foram controladas no desempenho da amostra:

Algumas variáveis como idade (6 / 16 anos), sexo (feminino / masculino) repetência (sem / com), local (centro / periferia) e tipo da escola (privada / pública) e, ainda, etnia (branco / não branco) foram estudadas, concluindo-se que algumas delas são relevantes na determinação dos resultados no conjunto verbal do teste WISC-IIl. A tabela 5 evidencia os coeficientes betas da Análise de Regressão, que determinam a relevância de cada variável em cada subteste e no total do conjunto verbal, assim como mostra, também, os valores dos coeficientes de determinação (R2) que indicam o quanto o modelo (conjunto de variáveis) explica os resultados. 
Tabela 5. Valores do beta e dos coeficientes de determinação.

\begin{tabular}{c|cccccc|c}
\hline Subteste & Repetência & Idade & Sexo & Etnia & Local & Tipo & $\mathrm{R}^{2}$ \\
\hline Informação & $-0,19^{*}$ & $0,78^{*}$ & $0,14^{*}$ & $-0,12^{*}$ & & & 0,81 \\
Vocabulário & $-0,25^{*}$ & $0,71^{*}$ & $0,11^{*}$ & $-0,11^{*}$ & $-0,19^{*}$ & & 0,81 \\
Semelhanças & $-0,27^{*}$ & $0,53^{*}$ & & & & $-0,14^{*}$ & 0,72 \\
Aritmética & $-0,17^{*}$ & $0,65^{*}$ & & & $-0,17^{*}$ & & 0,63 \\
Compreensão & $-0,21^{*}$ & $0,75^{*}$ & & & & & 0,67 \\
Dígitos & $-0,28^{*}$ & $0,51^{*}$ & & & $-0,21^{*}$ & & 0,53 \\
Total de acertos & $-0,25^{*}$ & $0,77^{*}$ & $0,09^{*}$ & & $-0,16^{*}$ & $-0,08^{*}$ & 0,84 \\
\hline
\end{tabular}

* Valores de $\mathrm{p}<0.0001$

Fonte: Dados da pesquisa Influências socioculturais na inteligência verbal: uma análise fundamentada no teste WISC-lll. 1994.

Os resultados mostram que as variáveis repetência e idade exercem influência no desempenho enquanto o sexo e a etnia mostram os menores efeitos, confirmando dados da literatura. Também observa-se que o nível socioeconômico-cultural, avaliado pelo local da escola (centro / periferia) é responsável por parte da variabilidade dos resultados obtidos no teste.

\section{Análise teórica dos itens}

Em relação à análise da adequação dos itens, os mais criticados pelos peritos referiram-se aos subtestes de Informação e Vocabulário (ver tab. 6), tendo sido os que apresentaram maior número de sugestões para alteração, parecendo serem os mais carregados de influências socioculturais.

Tabela 6. Itens do subtestes verbais do WISC-IJI considerados inadequados à população brasileira

\begin{tabular}{cccc}
\hline \multirow{2}{*}{ Subteste } & \multirow{2}{*}{ Número de itens } & \multicolumn{2}{c}{ Itens inadequados } \\
\cline { 3 - 4 } & 30 & 8 & $\%$ \\
\hline Informação & 19 & 2 & 26,66 \\
Semelhanças & 19 & 0 & 10,52 \\
Aritmética & 30 & 7 & 0 \\
Vocabulário & 18 & 1 & 23,33 \\
Compreensão & 116 & 18 & 5,55 \\
\hline Total & & & 15,51 \\
\hline
\end{tabular}

Fonte: Análise de juízes

A partir das sugestões colhidas na análise teórica dos itens sintetizou-se algumas propostas a serem consideradas para reformulação das questões inicialmente traduzidas do teste original, as quais serão testadas na nova aplicação do instrumento.

\section{Conclusões}

A partir da análise psicométrica de dados referentes à aplicação piloto do conjunto verbal do WISC-III, conclui-se que o instrumento parece adequado para nossa cultura, desde que algumas alterações sejam efetuadas. O estudo, realizado com as variáveis independentes, sugere a 
necessidade de uma análise detalhada sobre o viés da cultura/nível socioeconômico, no teste WISCIII, quando aplicado no contexto brasileiro. Por tanto, ao ser dado continuidade à pesquisa de adaptação do WISC-III, através da TRI (Item Response Theory) que procede a análise dos itens em relação aos parâmetros de discriminação, de dificuldade e da resposta aleatória, será estabelecido o dif (differential item functioning) para cada item do teste para amostras culturalmente diferentes, possibilitando discriminar as questões com dificuldades desiguais para cada grupo.

As sugestões colhidas na análise teórica dos itens serão incluídas numa nova versão do instrumento que será aplicado a uma amostra de 600 sujeitos, com idades entre 6 a 16 anos, matriculados na rede de ensino público e privado. A previsão de término do trabalho está planejada para final de 1999, pretendendo-se definir os índices de validade e precisão do instrumento e, ainda, estabelecer normas provisórias para interpretação dos resultados, proporcionando uma técnica de avaliação intelectual atualizada e adequada às características socioeconômicas-culturais da população brasileira.

\section{Referências}

Almeida, L. (1996). Considerações em tomo da medida da inteligência. Em L. Pasquali (Org), Teoria $e$ métodos de medida em Ciências do Comportamento (pp. 199-224). Brasília: UnB/INEP.

Figueiredo, V. (1994). Influências socioculturais na inteligência verbal: uma análise fundamentada no teste WISC-IIl. Dissertação de Mestrado, Pontifícia Universidade Católica do Rio Grande do Sul, Porto Alegre.

Gibello, B. (1986). Definições, discussões, divergências. Em: B. Gibello. A criança com distúrbios de inteligência (Trad. L. Fisher). (pp. 28-42). Porto Alegre: Artes Médicas.

Hutz, C. \& Bandeira, D. (1993). Tendências contemporâneas no uso de testes, uma análise da literatura brasileira e internacional. Psicologia: Reflexão e Crítica. 6, (12), 85-10 1.

Matarazzo,1. (1976). Medida e avaliação da inteligência do adulto (Trad. U. Arantes). São Paulo: Mariole.

Nascimento, E. (no prelo). Escala Wechsler de Inteligência para adultos- WAIS-/II: Novas perspectivas. Anais do IV Encontro Mineiro O Uso dos Testes Psicológicos. Belo Horizonte.

Paine, P. \& Lemgruber, V. (1978). Escala verbal do WISC: análise fatorial de uma amostra brasileira. InteramericanJournalofPsychology.12, 165-169.

Paine, P. \& Lemgruber, V. (1981). Adaptação brasileira da escala verbal do WISC. Arquivos brasileiros de psicologia. 33 (1-2), 32-56.

Safra, G. (1987). A escala Wechsler para crianças WISC. Em: M. Lopes (Org). Avaliação da inteligência (106-154). São Paulo: E.P.U.

Wechsler, D. (1991). Wechsler intelligence scale for children. - Third editíon (WISC-II/): Manual. San Antonio: Psychological Corporation.

Zachary, R. A. (1990). Wechsler InteIligence Scales: Theoretical and Practical considerations. Journal of Psychoeducational Assessment. 8, 276-289.

Zimmerman, 1., Gasser, A. (1977). Interpretación clinica de Ia Escala de inteligência de Wechsler para Ninos (WISC). (Trad. M. Benedet). Madrid: Tea. 